INPLASY

PROTOCOL

To cite: Zhang et al. Common Risk Factors for Postoperative Delirium in Elderly Patients with Different Surgical Procedures: a Systematic Review and Metaanalysis of Large Cohort Studies. Inplasy protocol 2021120119. doi: 10.37766/inplasy2021.12.0119

Received: 26 December 2021

Published: 26 December 2021

Corresponding author:

Dandan Zhang

1032691044@qq.com

Author Affiliation:

Shantou Central Hospital

Support: None.

Review Stage at time of this submission: Preliminary searches.

Conflicts of interest: None declared.

\section{Common Risk Factors for Postoperative Delirium in Elderly Patients with Different Surgical Procedures: a Systematic Review and Meta-analysis of Large Cohort Studies}

Zhang, DD1; Tang, SL2; Zeng, ZY3; Zhang, P4.

Review question / Objective: At present, the systematic reviews on the risk factors of postoperative delirium are mostly limited to a certain specialist field, such as orthopaedics, gastrointestinal surgery, cardiac surgery, vascular surgery and so on, and there are many research results on the risk factors. We summarized the items on the risk factors of postoperative delirium in guidelines, consensus and practical recommendations, such as the American Society for Enhanced Recovery and the Perioperative Quality Initiative (ASER/POQI) in 2020, the European Society of Anaesthesiology (ESA) in 2017, and the American Geriatrics Society (AGS) in 2014. The contents include more than 60 items. And, most of the risk factors do not have the evidence level and recommendation strength. Therefore, it is necessary to meta-analyze the risk factors that are common to different surgeries and have stronger predictive ability. The risk factors with universality and stronger prediction ability can be used to build a wider range of delirium risk prediction tool, which can be verified and promoted more widely.

INPLASY registration number: This protocol was registered with the International Platform of Registered Systematic Review and Meta-Analysis Protocols (INPLASY) on 26 December 2021 and was last updated on 26 December 2021 (registration number INPLASY2021120119).

\section{INTRODUCTION}

Review question / Objective: At present, the systematic reviews on the risk factors of postoperative delirium are mostly limited to a certain specialist field, such as orthopaedics, gastrointestinal surgery, cardiac surgery, vascular surgery and so on, and there are many research results on the risk factors. We summarized the items on the risk factors of postoperative delirium in guidelines, consensus and practical recommendations, such as the American Society for Enhanced Recovery and the Perioperative Quality Initiative (ASER/POQI) in 2020, the European Society of Anaesthesiology (ESA) in 2017, and the American Geriatrics Society (AGS) in 2014. 
The contents include more than 60 items. And, most of the risk factors do not have the evidence level and recommendation strength. Therefore, it is necessary to meta-analyze the risk factors that are common to different surgeries and have stronger predictive ability. The risk factors with universality and stronger prediction ability can be used to build a wider range of delirium risk prediction tool, which can be verified and promoted more widely.

Condition being studied: Delirium is a common clinical syndrome characterised by disturbed consciousness, cognitive function or perception, which has an acute onset and fluctuating course. Delirium can initiate a cascade of deleterious clinical events, including other major postoperative complications, prolonged hospitalization, loss of functional independence, reduced cognitive function, and death. Postoperative delirium (POD) often starts in the recovery room and occurs up to 5 days after surgery. There are few effective treatments for established delirium, but POD and its associated effects can be reduced by one-third with prevention strategies and early recognition. Risk factors for delirium need to be understood before implementing prevention strategies. At present, the research on its risk factors is mostly limited to a particular specialty. The purpose of this study is to develop an evidence-based list of risk factors that can be used by clinical staff in different departments and have stronger predictive power.

\section{METHODS}

Search strategy: From inception to October 2021, the cohort studies on risk factors of postoperative delirium in different surgical departments in PubMed and Web of science databases were systematically searched, and the sample size was $\geq 500$ cases. No language restrictions. The PubMed search was as follows: : [(MeSH terms "delirium") OR (Title/Abstract "delirium, confusion , confusion*, disorientation, bewilderment")] AND [(MeSH terms "Postoperative Period") OR
(Title/Abstract "postoperative, postoperative period, postoperative period*, post surgical, postsurgical, anesthesia recovery period, anesthesia recovery period* ,postanesthesia")] AND [(MeSH term "Risk Factors")OR (Title/ Abstract "risk factor* ,risk assessment")] The Web of science search was as follows: TOPIC: (delirium) AND TOPIC: (postoperativ e OR postoperative period ${ }^{\star}$ OR post suigical OR postsurgical OR surgery past) AND TOPIC: (risk factor* OR risk assessment).

Participant or population: Elderly patients who underwent elective or emergency surgery, aged $\geq 60$ years oldExclusion: Intensive care-only patients

Intervention: Exposures: The risk factors of postoperative delirium include preoperative, intraoperative and postoperative stages.Examples include:Preoperative:History of delirium, Cognitive impairment, Baseline dementia, Age, Partial dependence, malnutrition, $A S A \geq 3$, Depression, Opioid use, Education, Living alone, and so on. Intraoperative: Blood transfusion, Blood loss, Operation duration, and so on.Postoperative:ICU admission, Pain, Sleep disorders, Infection, and so on.

\section{Comparator: A non-exposed control} group: A group of elderly surgical patients without postoperative delirium.

Study designs to be included: Cohort Studies.

Eligibility criteria: The inclusion criteria are: (1) elderly patients who undergo elective or emergency surgery, age $\geq 60$ years old;(2) risk factors associate with postoperative delirium;(3) cohort study;(4) the main outcome is the occurrence of postoperative delirium, which is defined as the diagnosis of POD by effective delirium assessment tools, POD determined by linkage data such as ICD coding, and POD determined by chart review or effective recording;(5) sample size $\geq 500$ cases. The exclusion criteria are:(1) intensive care-only 
patients;(2) studies with incomplete reports on risk factors;(3) participants are not just elderly patients;(4) studies with low quality evaluation; case series, dissertations, book chapters, agreement articles, reviews, news articles, conference abstracts, letters to editors, editorials and review publications.

Information sources: We searched PubMed and Web of Science, restricting our query to publications with abstracts available from inception to October 2021, without language restrictions.

Main outcome(s): The outcome is the occurrence of postoperative delirium

(POD), which is defined as the diagnosis of POD by effective delirium assessment tools, POD determined by linkage data such as ICD coding, and POD determined by chart review or effective recording.

\section{Additional outcome(s): None.}

Data management: Study selection - Two authors (DDZ and ZYZ) will screen all studies. DDZ will screen all citations retrieved from the database searches, and both authors will evaluate the eligibility of the full text articles. Researchers will be blinded to each other's' decisions. Disagreements will be resolved by discussion or in consultation with a third author (SLT). Data extraction Two authors (DDZ and ZYZ), will abstract data using standardized forms. The first table extracts basic information about the study, including title, first author, year of publication, country, study design, age, sample size, type of surgery, delirium assessment tool, and univariate significant risk factors associated with POD. The second table extracts descriptive data (eg, mean and SD/event rates) for the occurrence and absence of postoperative delirium in the presence of each specific risk factor. Researchers will be blinded to each other's' decisions. Disagreements will be resolved by discussion or in consultation with a third author (SLT).
Quality assessment / Risk of bias analysis: The Newcastle-Ottawa Scale (NOS) will be used to assess the risk of bias in each cohort study. NOS consists of three parts, including patient selection, comparability of groups, and assessment of outcome. The quality of each study was graded as either level 1 ( 0 to 5 ) or level 2 (6 to 9). The quality of all papers will be evaluated by two independent authors (DDZ and ZYZ). Researchers will be blinded to each other's' decisions. Differences of opinion are resolved by consensus with the third author (SLT). When the number of included studies $\geq 10$, a funnel plot will be used to evaluate the existence of publication bias.

Strategy of data synthesis: In order to obtain more universal risk factors, first of all, we will include large sample studies, and secondly, in all the large sample studies, the univariate statistical results of at least two studies showed that a risk factor was a significant risk factor $(P<$ $0.05)$, then the risk factor will be included and will be considered to be a specific risk factor in this meta-analysis. Because the number and type of covariates used within risk factor analyses varied greatly across studies, in order to improve the consistency within our analysis, only univariate (or unadjusted) data in cohort studies will be extracted. For each specific risk factor, descriptive data (eg, mean and standard deviation (SD)/ event rate) included in each study will be extracted. When data were reported and extracted as median and interquartile range values, they will be converted to mean and SD values. We will generate the absolute rate and relative risk of postoperative delirium in elderly patients in the presence and absence of risk factors. For the pooled relative risk of the specific risk factors, Higgins $I^{2}$ values will be used to assess heterogeneity. When $I^{2} 0.1$ indicates that there is no heterogeneity among the results of each independent study, fixed-effect model will be used to pool data. When $I^{2} \geq 50$ and $P \leq 0.1$, sensitivity analysis or subgroup analysis will be used to identify the source of heterogeneity. If the heterogeneity could not be eliminated, random-effect model will be used to pool 
data. For the calculated pooled event rates for each specific risk factor, random-effect models will be used to describe the heterogeneity.

Subgroup analysis: We plan to conduct subgroup analysis for different types of study design and different surgical specialties. Because prospective cohort studies and retrospective cohort studies, as well as different surgical specialties, may bring some bias and heterogeneity, it is necessary to discuss them separately. Each group will include study design such as prospective/retrospective cohort study and surgical specialty such as hip surgery/ urology surgery/abdominal surgery/cardiac surgery.

Sensitivity analysis: We plan to remove an outlier for sensitivity analysis.

Language: No.

Country(ies) involved: China.

Keywords: Risk factors, postoperative delirium, elderly patients, meta-analysis, surgical procedures

Contributions of each author:

Author 1 - Dandan Zhang - The author contributes to draft the manuscript.

Email: 1032691044@qq.com

Author 2 - Shunluan Tang - The author contributes to evaluate the development of the selection criteria, and the risk of bias assessment strategy.

Email: 191888546@qq.com

Author 3 - Zeyin Zeng - The author contributes to evaluate the development of the selection criteria, and the risk of bias assessment strategy.

Email: 522214526@qq.com

Author 4 - Ping Zhang - The author reads, provides feedback and approves the final manuscript.

Email: 44729293@qq.com 